\title{
Modelling Fractal Waves on Shallow Water Surfaces via Local Fractional Korteweg-de Vries Equation
}

\author{
Xiao-Jun Yang, ${ }^{1}$ Jordan Hristov, ${ }^{2}$ H. M. Srivastava, ${ }^{3}$ and Bashir Ahmad \\ ${ }^{1}$ Department of Mathematics and Mechanics, China University of Mining and Technology, Xuzhou, Jiangsu 221008, China \\ ${ }^{2}$ Department of Chemical Engineering, University of Chemical Technology and Metallurgy, Sofia, 8 Kliment Ohridsky Boulevard, \\ 1756 Sofia, Bulgaria \\ ${ }^{3}$ Department of Mathematics and Statistics, University of Victoria, Victoria, BC, Canada V8W 3R4 \\ ${ }^{4}$ Department of Mathematics, Faculty of Science, King Abdulaziz University, P.O. Box 80203, Jeddah 21589, Saudi Arabia
}

Correspondence should be addressed to Xiao-Jun Yang; dyangxiaojun@163.com

Received 15 May 2014; Accepted 29 May 2014; Published 12 June 2014

Academic Editor: Dumitru Baleanu

Copyright (C) 2014 Xiao-Jun Yang et al. This is an open access article distributed under the Creative Commons Attribution License, which permits unrestricted use, distribution, and reproduction in any medium, provided the original work is properly cited.

A mathematical model of fractal waves on shallow water surfaces is developed by using the concepts of local fractional calculus. The derivations of linear and nonlinear local fractional versions of the Korteweg-de Vries equation describing fractal waves on shallow water surfaces are obtained.

\section{Introduction}

The mathematical model of shallow water waves, conceived by Boussinesq [1], was rediscovered by Korteweg and de Vries [2]. It is commonly known as Korteweg-de Vries equation $(\mathrm{KdV})[2,3]$ and is given by

$$
\frac{\partial}{\partial t} \phi(x, t)+\frac{\partial^{3}}{\partial x^{3}} \phi(x, t)-6 \phi(x, t) \frac{\partial}{\partial x} \phi(x, t)=0 .
$$

Several versions of the KdV equations found in the literature are listed below.

(i) Generalized KdV equation (GKdV) [4]

$$
\frac{\partial}{\partial t} \phi(x, t)+\phi(x, t) \frac{\partial}{\partial x} \phi(x, t)-\frac{\partial^{5}}{\partial x^{5}} \phi(x, t)=0
$$

(ii) Generalized-generalized KdV equation (GGKdV) [5]

$$
\frac{\partial}{\partial t} \phi(x, t)+\left(\frac{\partial}{\partial x} \phi(x, t)\right)^{3}+\frac{\partial}{\partial x} f(\phi)=0 .
$$

(iii) Deformed KdV equation (DKdV) [6]

$$
\begin{aligned}
\frac{\partial}{\partial t} \psi(x, t) & +\frac{\partial}{\partial x} \\
\times\left(\frac{\partial^{2}}{\partial x^{2}} \psi(x, t)-2 \eta \psi^{3}(x, t)\right. & \left.-\frac{3}{2} \frac{\psi(x, t)(\partial \psi(x, t) / \partial x)^{2}}{\eta+\psi^{2}(x, t)}\right)=0 .
\end{aligned}
$$

(iv) Modified-modified KdV equation (MM KdV) [6]

$$
\begin{aligned}
\frac{\partial}{\partial t} \phi(x, t) & +\frac{\partial^{3}}{\partial x^{3}} \phi(x, t)-\frac{1}{8}\left(\frac{\partial}{\partial x} \phi(x, t)\right)^{3} \\
& +\frac{\partial}{\partial x} \phi(x, t)\left(A e^{\mu}+B+C e^{-\mu}\right)=0,
\end{aligned}
$$

where $\eta, A, B$, and $C$ are constants.

(i) Modified KdV equation (M KdV) [7]

$$
\frac{\partial}{\partial t} \phi(x, t)+\frac{\partial^{3}}{\partial x^{3}} \phi(x, t) \pm 6 \phi^{2}(x, t) \frac{\partial}{\partial x} \phi(x, t)=0 .
$$


(ii) Spherical KdV (SKdV) was [7]

$$
\begin{aligned}
\frac{\partial}{\partial t} \psi(x, t)+\frac{\partial^{3}}{\partial x^{3}} \psi(x, t)-6 \psi(x, t) \frac{\partial}{\partial x} \psi(x, t) \\
+\frac{\psi(x, t)}{t}=0 .
\end{aligned}
$$

(iii) Cylindrical $\mathrm{KdV}$ equation $(\mathrm{CKdV})$ was [8]

$$
\begin{aligned}
\frac{\partial}{\partial t} \psi(x, t)+\frac{\partial^{3}}{\partial x^{3}} \psi(x, t)-6 \psi(x, t) \frac{\partial}{\partial x} \psi(x, t) \\
+\frac{\psi(x, t)}{2 t}=0 .
\end{aligned}
$$

For Further versions of KdV equation, we refer the reader to [9-12] and the references cited therein.

Recently, the fractional KdV equations have been discussed by several authors. Momani et al. [13] studied the KdV equation with both space- and time-fractional derivatives, while the time-fractional derivative case has been considered by El-Wakil et al. [14]. Atangana and Secer [15] developed solutions for coupled Korteweg-de Vries equations with timefractional derivatives [15]. Abdulaziz et al. [16] discussed the modified KdV equations with different space- and timefractional derivatives.

It is imperative to note that the above mentioned works are based on the fractional calculus of differentiable functions. However, there are certain nondifferentiable physical quantities describing the physical parameters locally, where the concept of differentiable functions is not applicable. In such cases the local fractional calculus (LFC) concept allows to obtain solutions adequate to such nondifferentiable problems [17-25] such as local fractional Helmholtz and diffusion equations [19], local fractional Navier-Stokes equations in fractal domain [21], local fractional Poisson and Laplace equations arising in the electrostatics in fractal domain [23], fractional models in forest gap [24], inhomogeneous local fractional wave equations [25], local fractional heat conduction equation [26], and other results [26-30].

In the present work, we focus on the derivation of the linear and the nonlinear local fractional versions of the Korteweg-de Vries equation describing fractal waves on shallow water surfaces.

The paper is organized as follows. In Section 2, we recall the local fractional conservation laws for the quantities in mathematical physics while the local fractional Kortewegde Vries equation is derived from local fractional calculus in Section 3. The conclusions are outlined in Section 4.

\section{Theoretical Background}

2.1. Local Fractional Conservation Laws Arising in Mathematical Physics. First of all, we discuss the local fractional conservation laws of mass, energy, and momentum in fractal media.

Let us consider the quantity $\psi(r, t)$ which varies within the fractal volume $V^{(\gamma)}$. Observe that the variations in $\psi(r, t)$ with respect to the fractal time corresponds to the variation in the flux through the fractal boundary $\mathbf{S}^{(\beta)}$ or by a source inside the volume $V^{(\gamma)}$. The integral form of local fractional conservation of the quantity $\psi(r, t)$ is given by $[17,19,21]$

$$
\begin{aligned}
& \frac{d^{\alpha}}{d t^{\alpha}} \iiint_{V^{(\gamma)}} \psi(r, t) d V^{(\gamma)} \\
& \quad=-\oiint_{S^{(\beta)}} \phi(r, t) \cdot d \mathbf{S}^{(\beta)}+\iiint_{V^{(\gamma)}} H(r, t) d V^{(\gamma)},
\end{aligned}
$$

where $\phi(r, t)=\psi(r, t) v(r, t)$ is the fractal flux vector and $H(r, t)$ is the source (sink) for a nondifferentiable quantity $\psi(r, t)$. 22]

The local fractional surface integral is defined by $[17,19-$

$$
\iint u\left(r_{P}\right) d \mathbf{S}^{(\beta)}=\lim _{N \rightarrow \infty} \sum_{P=1}^{N} u\left(r_{P}\right) \mathbf{n}_{P} \Delta S_{P}^{(\beta)}
$$

where $\Delta S_{P}^{(\beta)}$ is the local fractal surface and $N$ denote elements of the surface with unit normal local fractional vector $\mathbf{n}_{P}$. When $\Delta S_{P}^{(\beta)} \rightarrow 0$ as $N \rightarrow \infty$, the local fractional volume integral of the function $\mathbf{u}$ takes the form $[17,19-23]$

$$
\iiint \mathbf{u}\left(r_{P}\right) d V^{(\gamma)}=\lim _{N \rightarrow \infty} \sum_{P=1}^{N} \mathbf{u}\left(r_{P}\right) \Delta V_{P}^{(\gamma)}
$$

The local fractional derivative of a function $f(x)$ of order $\alpha$ is defined by $[17,24,25]$

$$
\frac{d^{\alpha} f\left(x_{0}\right)}{d x^{\alpha}}=\frac{\Delta^{\alpha}\left(f(x)-f\left(x_{0}\right)\right)}{\left(x-x_{0}\right)^{\alpha}},
$$

with

$$
\Delta^{\alpha}\left(f(x)-f\left(x_{0}\right)\right) \cong \Gamma(1+\alpha)\left[f(x)-f\left(x_{0}\right)\right] .
$$

Using (9), the local fractional differential form of the local fractional conservation balance of the quantity $\psi(r, t)$ can be expressed as

$$
\frac{\partial^{\alpha} \psi(r, t)}{\partial t^{\alpha}}+\nabla^{\alpha} \cdot \phi(r, t)=H(r, t) .
$$

The local fractional gradient of the scale function $\varphi$ emerging from (14) is [17]

$$
\nabla^{\alpha} \varphi=\lim _{d V^{(\gamma)} \rightarrow 0}\left(\frac{1}{d V^{(\gamma)}} \oiint_{S^{(\beta)}} \varphi d \mathbf{S}^{(\beta)}\right) .
$$

In the Cantorian coordinates, the local fractional conservation equation (14) with respect to $\psi(x, y, z, t)$ can be written as

$$
\frac{\partial^{\alpha} \psi(x, y, z, t)}{\partial t^{\alpha}}+\nabla^{\alpha} \cdot \phi(x, y, z, t)=H(x, y, z, t) .
$$


Alternatively

$$
\begin{gathered}
\frac{\partial^{\alpha} \psi(x, y, z, t)}{\partial t^{\alpha}}+\frac{\partial^{\alpha} \phi_{1}(x, y, z, t)}{\partial x^{\alpha}}+\frac{\partial^{\alpha} \phi_{2}(x, y, z, t)}{\partial y^{\alpha}} \\
+\frac{\partial^{\alpha} \phi_{3}(x, y, z, t)}{\partial z^{\alpha}}=H(x, y, z, t), \\
\phi(x, y, z, t)=\phi_{1}(x, y, z, t) i^{\alpha}+\phi_{2}(x, y, z, t) j^{\alpha} \\
+\phi_{3}(x, y, z, t) k^{\alpha} .
\end{gathered}
$$

Notice that the quantity $\phi(x, y, z, t)$ can represent mass, energy, or momentum in fractal media.

If $\rho$ denotes the fractal mass density, then the function $\phi=\rho \boldsymbol{v}$ is the mass fractal flux and $H(x, y, z, t)=0$. In this case, the local fractional conservation of mass in fractal media reads as

$$
\frac{\partial^{\alpha} \rho}{\partial t^{\alpha}}+\nabla^{\alpha} \cdot(\rho \boldsymbol{v})=0
$$

In passing $e$ remark that (18) is used to describe fractal physical problems $[17,19,20]$.

In the context of the present analysis, the local fractional conservation of energy $E$ in fractal media is

$$
\frac{\partial^{\alpha} E}{\partial t^{\alpha}}+\nabla^{\alpha} \cdot(E \boldsymbol{v})=H(x, y, z, t) .
$$

The function $\phi=E \boldsymbol{v}$ in (19) is the fractal flux vector of the energy in fractal media. Further, if the function $E=$ $\rho C_{\alpha} T$ denotes the amount of heat energy per unit fractal volume in fractal media, then the transport flux is

$$
\phi=\rho C T v .
$$

Thus, the conservation of thermal energy in fractal media can be expressed as

$$
\frac{\partial^{\alpha} \rho C_{\alpha} T}{\partial t^{\alpha}}+\nabla^{\alpha} \cdot\left(\rho C_{\alpha} T \boldsymbol{v}\right)=\nabla^{\alpha} \cdot k\left(\nabla^{\alpha} T\right)+H(x, y, z, t) .
$$

As a consequence of (21), the local fractional Fourier law (with fractal thermal conductivity $k$ ) reads as

$$
F=-k \nabla^{\alpha} T
$$

For constant $C_{\alpha}$ and $k,(21)$ can be rewritten as $[17,22]$

$$
\frac{\partial^{\alpha} T}{\partial t^{\alpha}}+v \cdot \nabla^{\alpha} T=a \nabla^{2 \alpha} T+\frac{H(x, y, z, t)}{\rho C_{\alpha}},
$$

where the fractal thermal diffusivity $a$ is

$$
a=\frac{k}{\rho C_{\alpha}} \text {. }
$$

The local fractional conservation of momentum in fractal media is

$$
\frac{\partial^{\alpha} M}{\partial t^{\alpha}}+\nabla^{\alpha} \cdot(M v)=H(x, y, z, t)
$$

where the quantity $M$ represents the momentum in fractal media while the function $\phi=M v$ is the fractal momentum flux vector fractal media.

If the momentum per unit fractal volume is $M=\rho \boldsymbol{v}$, then the sources due to fractal stresses and fractal body forces (gravity generated) are $\nabla^{\alpha} \cdot \theta$ and $H(x, y, z, t)=\rho g$, respectively. With this terminology, we have

$$
\frac{\partial^{\alpha} \rho \boldsymbol{v}}{\partial t^{\alpha}}+\nabla^{\alpha} \cdot(\rho \boldsymbol{v} \boldsymbol{v})=\nabla^{\alpha} \cdot \theta+\rho \mathbf{g}
$$

In view of the local fractional conservation of mass (18), (26) takes the form

$$
\frac{\partial^{\alpha} \boldsymbol{v}}{\partial t^{\alpha}}+\left(\boldsymbol{v} \cdot \nabla^{\alpha}\right) \boldsymbol{v}=\frac{1}{\rho} \nabla^{\alpha} \cdot \theta+\mathbf{g} .
$$

In $(26)\left(v \cdot \nabla^{\alpha}\right) v$ is the nondifferentiable advection of momentum in fractal media.

For compressible fluids, the general form of the NavierStokes equation on Cantor sets is [21]

$$
\begin{aligned}
\rho \frac{\partial^{\alpha} \boldsymbol{v}}{\partial t^{\alpha}}= & -\nabla^{\alpha} p+\frac{1}{3} \mu \nabla^{\alpha}\left(\left(\nabla^{\alpha} \cdot \boldsymbol{v}\right)\right)+\mu \nabla^{2 \alpha} \boldsymbol{v}+\rho \mathbf{b} \\
& -\rho \boldsymbol{v}\left(\nabla^{\alpha} \cdot \boldsymbol{v}\right)
\end{aligned}
$$

where $v$ is the fractal fluid velocity, $\mu$ is the dynamic viscosity, $p$ is the thermodynamic pressure, and $\mathbf{b}$ denotes the specific fractal body force.

If the term $(1 / 3) \mu \nabla^{\alpha}\left(\left(\nabla^{\alpha} \cdot v\right)\right)+\mu \nabla^{2 \alpha} v$ is zero, then (28) reduces to

$$
\rho \frac{\partial^{\alpha} \boldsymbol{v}}{\partial t^{\alpha}}=-\nabla^{\alpha} p+\rho \mathbf{b}-\rho \boldsymbol{v}\left(\nabla^{\alpha} \cdot \boldsymbol{v}\right)
$$

which is known as Cauchy's equation of motion of flows on Cantor sets [21].

For

$$
\frac{1}{\rho} \nabla^{\alpha} \cdot \theta=-\frac{1}{\rho} \nabla^{\alpha} p, \quad \mathbf{b}=-\mathbf{g},
$$

the Navier-Stokes equation on Cantor sets for a compressible fluid becomes

$$
\frac{\partial^{\alpha} \boldsymbol{v}}{\partial t^{\alpha}}+\boldsymbol{v}\left(\nabla^{\alpha} \cdot \boldsymbol{v}\right)=-\frac{1}{\rho} \nabla^{\alpha} p-\mathbf{g} .
$$

\subsection{Fractal Water Waves}

2.2.1. Linear Theory for Fractal Water Waves. Let us consider the following local fractional conservation equations of fluid motion in fractal media (Cauchy's equation of motion of flows on Cantor sets):

$$
\begin{gathered}
\frac{\partial^{\alpha} \rho}{\partial t^{\alpha}}+\nabla^{\alpha} \cdot(\rho \boldsymbol{v})=0 \\
\frac{\partial^{\alpha} \boldsymbol{v}}{\partial t^{\alpha}}+\boldsymbol{v}\left(\nabla^{\alpha} \cdot \boldsymbol{v}\right)=-\frac{1}{\rho} \nabla^{\alpha} p-\mathbf{g} .
\end{gathered}
$$


If the fractal fluid is incompressible and locally fractional irrotational, then we have

$$
\begin{gathered}
\nabla^{\alpha} \rho=0, \\
\frac{\partial^{\alpha} \rho}{\partial t^{\alpha}}=0, \\
\nabla^{\alpha} \times \boldsymbol{v}=0 .
\end{gathered}
$$

From (32) and (37), we have

$$
\begin{gathered}
\boldsymbol{v}=\nabla^{\alpha} \varphi, \\
\nabla^{2 \alpha} \varphi=0 .
\end{gathered}
$$

The local fractional Laplace operator is

$$
\nabla^{\alpha} \cdot \nabla^{\alpha}=\nabla^{2 \alpha}
$$

We notice that (38) is the local fractional Laplace equation (see $[21,23])$.

If the following relationship is valid [21]

$$
\boldsymbol{v} \cdot \nabla^{\alpha} \boldsymbol{v}=\nabla^{\alpha}\left(\frac{\boldsymbol{v} \cdot \boldsymbol{v}}{2}\right)-\left(\nabla^{\alpha} \times \boldsymbol{v}\right) \times \boldsymbol{v}
$$

Then, we have

$$
\boldsymbol{v} \cdot \nabla^{\alpha} \boldsymbol{v}=\nabla^{\alpha}\left(\frac{\boldsymbol{v} \cdot \boldsymbol{v}}{2}\right)
$$

Hence, from (33) and (41), we get

$$
\frac{\partial^{\alpha}}{\partial t^{\alpha}}\left(\nabla^{\alpha} \varphi\right)+v\left(\nabla^{\alpha} \cdot v\right)=-\frac{1}{\rho} \nabla^{\alpha} p-\mathbf{g},
$$

which leads to

$$
\frac{\partial^{\alpha}}{\partial t^{\alpha}}\left(\nabla^{\alpha} \varphi\right)+\nabla^{\alpha}\left(\frac{\boldsymbol{v} \cdot \boldsymbol{v}}{2}\right)=-\frac{1}{\rho} \nabla^{\alpha} p-\mathbf{g} .
$$

Equation (43) can be rewritten in terms of local fractional gradient as

$$
\nabla^{\alpha}\left(\frac{\partial^{\alpha} \varphi}{\partial t^{\alpha}}+\frac{\boldsymbol{v} \cdot \boldsymbol{v}}{2}+\frac{p}{\rho}+\mathbf{g} y\right)=0
$$

or

$$
\nabla^{\alpha}\left(\frac{\partial^{\alpha} \varphi}{\partial t^{\alpha}}+\frac{\nabla^{\alpha} \varphi \cdot \nabla^{\alpha} \varphi}{2}+\frac{p}{\rho}+\mathbf{g} \frac{y^{\alpha}}{\Gamma(1+\alpha)}\right)=0 .
$$

From (45), we have

$$
\frac{\partial^{\alpha} \varphi}{\partial t^{\alpha}}+\frac{\nabla^{\alpha} \varphi \cdot \nabla^{\alpha} \varphi}{2}+\frac{p-p_{0}}{\rho}+\mathbf{g} y=n(t),
$$

where $p_{0}$ is the initial pressure.

Let us suggest that the velocity of the fractal flow normal to the fractal interface can be described as

$$
f(x, z, t)=\eta(x, z, t)-y
$$

and is equal to the velocity of the fractal interface normal to itself. With these suggestions, we obtain

$$
\frac{\partial^{\alpha} \eta}{\partial t^{\alpha}}+\nabla^{\alpha} \varphi \cdot \nabla^{\alpha} \eta=v_{y}
$$

which is the fractal kinematic equation on the fractal boundary with

$$
\boldsymbol{v}_{y}=\frac{\partial^{\alpha} \varphi}{\partial y^{\alpha}}
$$

When the fractal boundary condition at the free surface is specified, then it follows from (46) and (48) that

$$
\begin{gathered}
\frac{\partial^{\alpha} \eta}{\partial t^{\alpha}}+\frac{\partial^{\alpha} \varphi}{\partial x^{\alpha}} \frac{\partial^{\alpha} \eta}{\partial x^{\alpha}}+\frac{\partial^{\alpha} \varphi}{\partial z^{\alpha}} \frac{\partial^{\alpha} \eta}{\partial z^{\alpha}}=\frac{\partial^{\alpha} \varphi}{\partial y^{\alpha}}, \\
\frac{\partial^{\alpha} \varphi}{\partial t^{\alpha}}+\frac{1}{2}\left(\frac{\partial^{\alpha} \varphi}{\partial x^{\alpha}} \frac{\partial^{\alpha} \varphi}{\partial x^{\alpha}}+\frac{\partial^{\alpha} \varphi}{\partial y^{\alpha}} \frac{\partial^{\alpha} \varphi}{\partial y^{\alpha}}+\frac{\partial^{\alpha} \varphi}{\partial z^{\alpha}} \frac{\partial^{\alpha} \varphi}{\partial z^{\alpha}}\right) \\
+\mathbf{g} \eta=0,
\end{gathered}
$$

where $p=p_{0}, n(t)=0$, and $\eta(x, z, t)=y$.

If the bottom section of the flow is considered, then

$$
y=-h_{0}(x, z) \text {. }
$$

Further, if the normal velocity of the flow is zero at the fixed solid boundary, (50) gives

$$
\frac{\partial^{\alpha} \varphi}{\partial y^{\alpha}}+\frac{\partial^{\alpha} \varphi}{\partial x^{\alpha}} \frac{\partial^{\alpha} h_{0}}{\partial x^{\alpha}}+\frac{\partial^{\alpha} \varphi}{\partial z^{\alpha}} \frac{\partial^{\alpha} h_{0}}{\partial z^{\alpha}}=0 .
$$

For a horizontal bottom, we have $y=-h_{0}(x, z)$ which leads to

$$
\frac{\partial^{\alpha} \varphi}{\partial y^{\alpha}}=0
$$

or

$$
\frac{\partial^{\alpha} \varphi}{\partial x^{\alpha}} \frac{\partial^{\alpha} h_{0}}{\partial x^{\alpha}}+\frac{\partial^{\alpha} \varphi}{\partial z^{\alpha}} \frac{\partial^{\alpha} h_{0}}{\partial z^{\alpha}}=0 .
$$

Therefore, at the free surface, we have

$$
\begin{gathered}
\frac{\partial^{\alpha} \eta}{\partial t^{\alpha}}=\frac{\partial^{\alpha} \varphi}{\partial y^{\alpha}}, \\
\frac{\partial^{\alpha} \varphi}{\partial t^{\alpha}}+\mathbf{g} \eta=0,
\end{gathered}
$$

where $\eta(x, z, t)=y$.

For $y=0$, we find from (56) and (57) that

$$
\frac{\partial^{2 \alpha} \varphi}{\partial t^{2 \alpha}}+\mathbf{g} \frac{\partial^{\alpha} \varphi}{\partial y^{\alpha}}=0 .
$$

Therefore, we define the line problem for a water wave as follows:

$$
\begin{gathered}
\frac{\partial^{2 \alpha} \varphi}{\partial x^{2 \alpha}}+\frac{\partial^{2 \alpha} \varphi}{\partial y^{2 \alpha}}+\frac{\partial^{2 \alpha} \varphi}{\partial z^{2 \alpha}}=0, \quad-h_{0}(x, z)<y<0 ; \\
\frac{\partial^{2 \alpha} \varphi}{\partial t^{2 \alpha}}+\mathbf{g} \frac{\partial^{\alpha} \varphi}{\partial y^{\alpha}}=0, \quad y=0 ; \\
\frac{\partial^{\alpha} \varphi}{\partial y^{\alpha}}+\frac{\partial^{\alpha} \varphi}{\partial x^{\alpha}} \frac{\partial^{\alpha} h_{0}}{\partial x^{\alpha}}+\frac{\partial^{\alpha} \varphi}{\partial z^{\alpha}} \frac{\partial^{\alpha} h_{0}}{\partial z^{\alpha}}=0, \quad y=-h_{0}(x, z) .
\end{gathered}
$$


From (57), we may present the fractal surface as

$$
\eta(x, z, t)=-\frac{1}{\mathbf{g}} \frac{\partial^{\alpha} \varphi(x, 0, z, t)}{\partial t^{\alpha}} .
$$

2.2.2. Nonlinear Theory of Fractal Water Waves. The linear wave equation given in [21] is

$$
\frac{\partial^{2 \alpha} \eta}{\partial t^{2 \alpha}}+\omega \frac{\partial^{2 \alpha} \eta}{\partial x^{2 \alpha}}=0
$$

where $\omega$ is a constant.

From (43), we get

$$
\frac{1}{\rho} \nabla^{\alpha} p-\mathbf{g}=0
$$

or

$$
\frac{1}{\rho} \frac{\partial^{\alpha} \eta}{\partial y^{\alpha}} p-\mathbf{g}=0
$$

Then

$$
p-p_{0}=\mathbf{g} \rho(\eta-y) .
$$

From (33) and (64), we have

$$
\frac{\partial^{\alpha} \boldsymbol{v}}{\partial t^{\alpha}}+\boldsymbol{v}\left(\nabla^{\alpha} \cdot \boldsymbol{v}\right)=-\mathbf{g} \nabla^{\alpha} \eta
$$

Consequently

$$
\begin{aligned}
& \frac{\partial^{2 \alpha} \varphi}{\partial t^{\alpha} \partial x^{\alpha}}+ \frac{\partial^{\alpha} \varphi}{\partial x^{\alpha}} \frac{\partial^{2 \alpha} \varphi}{\partial x^{2 \alpha}}+\frac{\partial^{\alpha} \varphi}{\partial y^{\alpha}} \frac{\partial^{2 \alpha} \varphi}{\partial y^{\alpha} \partial x^{\alpha}} \\
&+\frac{\partial^{\alpha} \varphi}{\partial z^{\alpha}} \frac{\partial^{2 \alpha} \varphi}{\partial z^{\alpha} \partial x^{\alpha}}=-\mathbf{g} \frac{\partial^{\alpha} \eta}{\partial x^{\alpha}}, \\
& \frac{\partial^{2 \alpha} \varphi}{\partial t^{\alpha} \partial z^{\alpha}}+\frac{\partial^{\alpha} \varphi}{\partial x^{\alpha}} \frac{\partial^{2 \alpha} \varphi}{\partial x^{\alpha} \partial z^{\alpha}}+\frac{\partial^{\alpha} \varphi}{\partial y^{\alpha}} \frac{\partial^{2 \alpha} \varphi}{\partial y^{\alpha} \partial z^{\alpha}} \\
&+\frac{\partial^{\alpha} \varphi}{\partial z^{\alpha}} \frac{\partial^{2 \alpha} \varphi}{\partial z^{2 \alpha}}=-\mathbf{g} \frac{\partial^{\alpha} \eta}{\partial z^{\alpha}} .
\end{aligned}
$$

If the conditions $\left(\partial^{2 \alpha} \varphi / \partial y^{\alpha} \partial z^{\alpha}\right)=0$ and $\left(\partial^{2 \alpha} \varphi /\right.$ $\left.\partial y^{\alpha} \partial x^{\alpha}\right)=0$ are satisfied, then from (66), we get

$$
\begin{gathered}
\frac{\partial^{2 \alpha} \varphi}{\partial t^{\alpha} \partial x^{\alpha}}+\frac{\partial^{\alpha} \varphi}{\partial x^{\alpha}} \frac{\partial^{2 \alpha} \varphi}{\partial x^{2 \alpha}}+\frac{\partial^{\alpha} \varphi}{\partial z^{\alpha}} \frac{\partial^{2 \alpha} \varphi}{\partial z^{\alpha} \partial x^{\alpha}}=-\mathbf{g} \frac{\partial^{\alpha} \eta}{\partial x^{\alpha}}, \\
\frac{\partial^{2 \alpha} \varphi}{\partial t^{\alpha} \partial z^{\alpha}}+\frac{\partial^{\alpha} \varphi}{\partial x^{\alpha}} \frac{\partial^{2 \alpha} \varphi}{\partial x^{\alpha} \partial z^{\alpha}}+\frac{\partial^{\alpha} \varphi}{\partial z^{\alpha}} \frac{\partial^{2 \alpha} \varphi}{\partial z^{2 \alpha}}=-\mathbf{g} \frac{\partial^{\alpha} \eta}{\partial z^{\alpha}} .
\end{gathered}
$$

Further, from (32) and (35), we obtain

$$
\frac{\partial^{\alpha} h}{\partial t^{\alpha}}+\nabla^{\alpha} \cdot(h v)=0
$$

where

$$
h=h_{0}+\eta \text {. }
$$

Using (51), (68), and (69), we obtain the local fractional conservation equations for one-dimensional waves on the bottom given by

$$
\begin{gathered}
\frac{\partial^{\alpha}}{\partial t^{\alpha}}\left(\frac{\partial^{\alpha} \varphi}{\partial x^{\alpha}}\right)+\frac{\partial^{\alpha} \varphi}{\partial x^{\alpha}} \frac{\partial^{2 \alpha} \varphi}{\partial x^{2 \alpha}}+\mathbf{g} \frac{\partial^{\alpha} h}{\partial x^{\alpha}}=0, \\
\frac{\partial^{\alpha} h}{\partial t^{\alpha}}+\frac{\partial^{\alpha}}{\partial x^{\alpha}}\left(h \frac{\partial^{\alpha} \varphi}{\partial x^{\alpha}}\right)=0,
\end{gathered}
$$

which lead to

$$
\begin{aligned}
& \frac{\partial^{\alpha}}{\partial t^{\alpha}}\left(h \frac{\partial^{\alpha} \varphi}{\partial x^{\alpha}}\right)+\frac{\partial^{\alpha}}{\partial x^{\alpha}}\left(\left(\frac{\partial^{\alpha} \varphi}{\partial x^{\alpha}}\right)^{2} h+\frac{1}{2} \mathbf{g} h^{2}\right)=0, \\
& \frac{\partial^{\alpha}}{\partial t^{\alpha}}\left(\frac{1}{2}\left(\frac{\partial^{\alpha} \varphi}{\partial x^{\alpha}}\right)^{2} h+\frac{1}{2} \mathbf{g} h^{2}\right) \\
&+\frac{\partial^{\alpha}}{\partial x^{\alpha}}\left(\frac{1}{2}\left(\frac{\partial^{\alpha} \varphi}{\partial x^{\alpha}}\right)^{3} h+\frac{1}{2} \mathbf{g} h^{2} \frac{\partial^{\alpha} \varphi}{\partial x^{\alpha}}\right)=0 .
\end{aligned}
$$

Furthermore, from (38), (50), (51), and (59), we get

$\frac{\partial^{2 \alpha} \varphi}{\partial x^{2 \alpha}}+\frac{\partial^{2 \alpha} \varphi}{\partial y^{2 \alpha}}=0, \quad-\infty<x<+\infty, 0 \leq y \leq \eta(x, t)$,

$$
\begin{gathered}
\frac{\partial^{\alpha} \eta}{\partial t^{\alpha}}+\frac{\partial^{\alpha} \varphi}{\partial x^{\alpha}} \frac{\partial^{\alpha} \eta}{\partial x^{\alpha}}=\frac{\partial^{\alpha} \varphi}{\partial y^{\alpha}}, \quad y=1+\varsigma \eta, \\
\frac{\partial^{\alpha} \varphi}{\partial t^{\alpha}}+\frac{1}{2}\left(\frac{\partial^{\alpha} \varphi}{\partial x^{\alpha}} \frac{\partial^{\alpha} \varphi}{\partial x^{\alpha}}+\frac{\partial^{\alpha} \varphi}{\partial y^{\alpha}} \frac{\partial^{\alpha} \varphi}{\partial y^{\alpha}}\right)+\mathbf{g} \eta=0,
\end{gathered}
$$

with

$$
\frac{\partial^{\alpha} \varphi}{\partial y^{\alpha}}=0, \quad y=0
$$

\section{Local Fractional Korteweg-de Vries Equation}

Using (74) and (76), it is possible to expand the fractal velocity potential into a nondifferentiable series with respect to $y$ in the following form:

$$
\varphi(x, y, t)=\sum_{i=0}^{\infty} \frac{y^{n \alpha}}{\Gamma(1+n \alpha)} \varphi_{n}(x, t) .
$$

Then, it follows from (74) and (78) that

$$
\frac{\partial^{2 \alpha} \varphi}{\partial x^{2 \alpha}}=\sum_{i=0}^{\infty} \frac{y^{n \alpha}}{\Gamma(1+n \alpha)} \frac{\partial^{2 \alpha} \varphi_{n}(x, t)}{\partial x^{2 \alpha}},
$$

$$
\frac{\partial^{2 \alpha} \varphi}{\partial y^{2 \alpha}}=\sum_{i=2}^{\infty} \frac{y^{(n-2) \alpha}}{\Gamma(1+(n-2) \alpha)} \varphi_{n}(x, t)
$$

$$
=\sum_{i=0}^{\infty} \Gamma(1+(n+2) \alpha) y^{n \alpha} \varphi_{n+2}(x, t) \text {. }
$$


Hence,

$$
\frac{\partial^{2 \alpha} \varphi_{n}(x, t)}{\partial x^{2 \alpha}}+\frac{\Gamma(1+(n+2) \alpha)}{\Gamma(1+n \alpha)} \varphi_{n+2}(x, t)=0,
$$

$\forall n \in N$

Thus, from (77), we get

$$
\begin{aligned}
\frac{\partial^{\alpha} \varphi}{\partial y^{\alpha}} & =\sum_{i=1}^{\infty} \frac{y^{(n-1) \alpha}}{\Gamma(1+(n-1) \alpha)} \varphi_{n}(x, t) \\
& =\varphi_{1}(x, t)=0 .
\end{aligned}
$$

Equations (70) and (73) lead to

$$
\begin{gathered}
n=0: \frac{\partial^{2 \alpha} \varphi_{0}(x, t)}{\partial x^{2 \alpha}}+\frac{\Gamma(1+(n+2) \alpha)}{\Gamma(1+n \alpha)} \varphi_{2}(x, t)=0, \\
n=1: \frac{\partial^{2 \alpha} \varphi_{1}(x, t)}{\partial x^{2 \alpha}}+\frac{\Gamma(1+(n+2) \alpha)}{\Gamma(1+n \alpha)} \varphi_{3}(x, t)=0, \\
n=2: \frac{\partial^{2 \alpha} \varphi_{2}(x, t)}{\partial x^{2 \alpha}}+\frac{\Gamma(1+(n+2) \alpha)}{\Gamma(1+n \alpha)} \varphi_{4}(x, t)=0, \\
n=3: \frac{\partial^{2 \alpha} \varphi_{3}(x, t)}{\partial x^{2 \alpha}}+\frac{\Gamma(1+(n+2) \alpha)}{\Gamma(1+n \alpha)} \varphi_{5}(x, t)=0, \\
n=4: \frac{\partial^{2 \alpha} \varphi_{4}(x, t)}{\partial x^{2 \alpha}}+\frac{\Gamma(1+(n+2) \alpha)}{\Gamma(1+n \alpha)} \varphi_{6}(x, t)=0, \\
n=k: \frac{\partial^{2 \alpha} \varphi_{k}(x, t)}{\partial x^{2 \alpha}}+\frac{\Gamma(1+(k+2) \alpha)}{\Gamma(1+k \alpha)} \varphi_{k+2}(x, t)=0 .
\end{gathered}
$$

Hence, we get

$$
\varphi(x, y, t)=\sum_{i=0}^{\infty} \frac{y^{2 i \alpha}}{\Gamma(1+2 i \alpha)}(-1)^{i} \frac{\partial^{2 i \alpha}}{\partial x^{2 i \alpha}} f\left(\varphi_{0}\right),
$$

where

$$
f\left(\varphi_{0}\right)=\varphi_{0}(x, t) .
$$

Similarly, from (84) and (85), we can get

$$
\begin{aligned}
\frac{\partial^{\alpha} \varphi}{\partial x^{\alpha}}= & \frac{\partial^{\alpha}}{\partial x^{\alpha}} f\left(\varphi_{0}\right)-\frac{y^{2 \alpha}}{\Gamma(1+2 \alpha)} \frac{\partial^{3 \alpha}}{\partial x^{3 \alpha}} f\left(\varphi_{0}\right)+O\left(x^{2 \alpha}\right), \\
\frac{\partial^{\alpha} \varphi}{\partial y^{\alpha}}= & -\frac{y^{\alpha}}{\Gamma(1+\alpha)} \frac{\partial^{2 \alpha}}{\partial x^{2 \alpha}} f\left(\varphi_{0}\right)+\frac{y^{3 \alpha}}{\Gamma(1+3 \alpha)} \frac{\partial^{4 \alpha}}{\partial x^{4 \alpha}} f\left(\varphi_{0}\right) \\
& +O\left(x^{2 \alpha}\right) .
\end{aligned}
$$

In order to obtain a dimensionless form of (74)-(77), we make the following scale transformations:

$$
\begin{gathered}
x \longrightarrow x \lambda, \\
y \longrightarrow h y, \\
\varphi \longrightarrow \frac{\varsigma \lambda^{\alpha} c_{0}^{\alpha} \varphi}{h^{\alpha}}, \\
t \longrightarrow \frac{t \lambda}{c_{0}}, \\
\eta \longrightarrow \varsigma \eta,
\end{gathered}
$$

so that

$$
\begin{gathered}
\varepsilon^{2 \alpha} \frac{\partial^{2 \alpha} \varphi}{\partial x^{2 \alpha}}+\frac{\partial^{2 \alpha} \varphi}{\partial y^{2 \alpha}}=0, \quad 0 \leq y \leq 1+a \eta, \\
\frac{\partial^{\alpha} \eta}{\partial t^{\alpha}}+a \frac{\partial^{\alpha} \varphi}{\partial x^{\alpha}} \frac{\partial^{\alpha} \eta}{\partial x^{\alpha}}-\frac{1}{\varepsilon^{2 \alpha}} \frac{\partial^{\alpha} \varphi}{\partial y^{\alpha}}=0, \quad y=1+a \eta, \\
\frac{\partial^{\alpha} \varphi}{\partial t^{\alpha}}+\frac{a}{2} \frac{\partial^{\alpha} \varphi}{\partial x^{\alpha}} \frac{\partial^{\alpha} \varphi}{\partial x^{\alpha}}+\frac{1}{2} \frac{a}{\varepsilon^{2 \alpha}} \frac{\partial^{\alpha} \varphi}{\partial y^{\alpha}} \frac{\partial^{\alpha} \varphi}{\partial y^{\alpha}}+\eta=0, \\
y=1+a \eta, \\
\frac{\partial^{\alpha} \varphi}{\partial y^{\alpha}}=0, \quad y=0 .
\end{gathered}
$$

In this context, the equation for the free water surface is

$$
y=1+a \eta,
$$

Here $c_{0}=\sqrt{g h}$ is the linear wave velocity in shallow water. The two small parameters are $a=\varsigma / h^{\alpha}$ and $\varepsilon=$ $h / \lambda$ with depth of the water $h$, while $\varsigma$ and $\lambda$ are the typical height and length of the solitary wave, respectively

Equations (84), (88), and (91) allow developing a nondifferentiable series with respect to $\varepsilon$ in the form

$$
\varphi(x, \eta, t)=\sum_{i=0}^{\infty} \frac{y^{2 i \alpha}}{\Gamma(1+2 i \alpha)}(-1)^{i} \frac{\partial^{2 i \alpha}}{\partial x^{2 i \alpha}} f\left(\varphi_{0}\right) \varepsilon^{2 i \alpha} .
$$

In view of (84), (89), and (90), we have

$$
\begin{aligned}
\varphi= & f\left(\varphi_{0}\right)-\frac{y^{2 \alpha}}{\Gamma(1+2 \alpha)} \frac{\partial^{2 \alpha}}{\partial x^{2 \alpha}} f\left(\varphi_{0}\right) \varepsilon^{2 \alpha} \\
& +\frac{y^{4 \alpha}}{\Gamma(1+4 \alpha)} \frac{\partial^{4 \alpha}}{\partial x^{4 \alpha}} f\left(\varphi_{0}\right) \varepsilon^{4 \alpha}+O\left(\varepsilon^{4 \alpha}\right),
\end{aligned}
$$

so that

$$
\begin{aligned}
\frac{\partial^{\alpha} \varphi}{\partial x^{\alpha}}= & \frac{\partial^{\alpha} f\left(\varphi_{0}\right)}{\partial x^{\alpha}}-\frac{(1+a \eta)^{2 \alpha}}{\Gamma(1+2 \alpha)} \frac{\partial^{3 \alpha}}{\partial x^{3 \alpha}} f\left(\varphi_{0}\right) \varepsilon^{2 \alpha} \\
& +\frac{(1+a \eta)^{4 \alpha}}{\Gamma(1+4 \alpha)} \frac{\partial^{5 \alpha}}{\partial x^{5 \alpha}} f\left(\varphi_{0}\right) \varepsilon^{4 \alpha}+O\left(\varepsilon^{4 \alpha}\right),
\end{aligned}
$$




$$
\begin{aligned}
\frac{\partial^{\alpha} \varphi}{\partial y^{\alpha}}= & -\frac{(1+a \eta)^{\alpha}}{\Gamma(1+\alpha)} \frac{\partial^{2 \alpha}}{\partial x^{2 \alpha}} f\left(\varphi_{0}\right) \varepsilon^{2 \alpha} \\
& +\frac{(1+a \eta)^{3 \alpha}}{\Gamma(1+3 \alpha)} \frac{\partial^{4 \alpha}}{\partial x^{4 \alpha}} f\left(\varphi_{0}\right) \varepsilon^{4 \alpha}+O\left(\varepsilon^{4 \alpha}\right),
\end{aligned}
$$

which leads to

$$
\begin{aligned}
& \frac{\partial^{\alpha} \eta}{\partial t^{\alpha}}+a\left(\frac{\partial^{\alpha} f\left(\varphi_{0}\right)}{\partial x^{\alpha}}-\frac{(1+a \eta)^{2 \alpha}}{\Gamma(1+2 \alpha)} \frac{\partial^{3 \alpha}}{\partial x^{3 \alpha}} f\left(\varphi_{0}\right) \varepsilon^{2 \alpha}\right. \\
& \left.+\frac{(1+a \eta)^{4 \alpha}}{\Gamma(1+4 \alpha)} \frac{\partial^{5 \alpha}}{\partial x^{5 \alpha}} f\left(\varphi_{0}\right) \varepsilon^{4 \alpha}\right) \frac{\partial^{\alpha} \eta}{\partial x^{\alpha}} \\
& -\frac{1}{\varepsilon^{2 \alpha}}\left(-\frac{(1+a \eta)^{\alpha}}{\Gamma(1+\alpha)} \frac{\partial^{2 \alpha}}{\partial x^{2 \alpha}} f\left(\varphi_{0}\right) \varepsilon^{2 \alpha}\right. \\
& \left.+\frac{(1+a \eta)^{3 \alpha}}{\Gamma(1+3 \alpha)} \frac{\partial^{4 \alpha}}{\partial x^{4 \alpha}} f\left(\varphi_{0}\right) \varepsilon^{4 \alpha}\right) \\
& +O\left(\varepsilon^{4 \alpha}\right)=0, \\
& \frac{\partial^{\alpha} f\left(\varphi_{0}\right)}{\partial t^{\alpha}}-\frac{(1+a \eta)^{2 \alpha}}{\Gamma(1+2 \alpha)} \frac{\partial^{2 \alpha}}{\partial x^{2 \alpha}} \frac{\partial^{\alpha} f\left(\varphi_{0}\right)}{\partial t^{\alpha}} \varepsilon^{2 \alpha} \\
& +\frac{(1+a \eta)^{4 \alpha}}{\Gamma(1+4 \alpha)} \frac{\partial^{4 \alpha}}{\partial x^{4 \alpha}} \frac{\partial^{\alpha}}{\partial t^{\alpha}} f\left(\varphi_{0}\right) \varepsilon^{4 \alpha} \\
& +\frac{a}{2}\left(\frac{\partial^{\alpha} f\left(\varphi_{0}\right)}{\partial x^{\alpha}}+\frac{(1+a \eta)^{4 \alpha}}{\Gamma(1+4 \alpha)} \frac{\partial^{5 \alpha}}{\partial x^{5 \alpha}} f\left(\varphi_{0}\right) \varepsilon^{4 \alpha}\right. \\
& \left.-\frac{(1+a \eta)^{2 \alpha}}{\Gamma(1+2 \alpha)} \frac{\partial^{3 \alpha}}{\partial x^{3 \alpha}} f\left(\varphi_{0}\right) \varepsilon^{2 \alpha}\right)^{2} \\
& +\frac{1}{2} \frac{a}{\varepsilon^{2 \alpha}}\left(-\frac{(1+a \eta)^{\alpha}}{\Gamma(1+\alpha)} \frac{\partial^{2 \alpha}}{\partial x^{2 \alpha}} f\left(\varphi_{0}\right) \varepsilon^{2 \alpha}\right. \\
& \left.+\frac{(1+a \eta)^{3 \alpha}}{\Gamma(1+3 \alpha)} \frac{\partial^{4 \alpha}}{\partial x^{4 \alpha}} f\left(\varphi_{0}\right) \varepsilon^{4 \alpha}\right)^{2} \\
& +\eta+O\left(\varepsilon^{4 \alpha}\right)=0 .
\end{aligned}
$$

Hence, from (97) and (98), we obtain

$$
\begin{aligned}
\frac{\partial^{\alpha} \eta}{\partial t^{\alpha}}+ & \left(a \frac{\partial^{\alpha} f\left(\varphi_{0}\right)}{\partial x^{\alpha}} \frac{\partial^{\alpha} \eta}{\partial x^{\alpha}}+\frac{(1+a \eta)^{\alpha}}{\Gamma(1+\alpha)} \frac{\partial^{2 \alpha}}{\partial x^{2 \alpha}} f\left(\varphi_{0}\right)\right) \\
- & \left\{\frac{(1+a \eta)^{2 \alpha}}{\Gamma(1+2 \alpha)} \frac{\partial^{3 \alpha}}{\partial x^{3 \alpha}} f\left(\varphi_{0}\right) a \frac{\partial^{\alpha} \eta}{\partial x^{\alpha}}\right. \\
& \left.+\frac{(1+a \eta)^{3 \alpha}}{\Gamma(1+3 \alpha)} \frac{\partial^{4 \alpha}}{\partial x^{4 \alpha}} f\left(\varphi_{0}\right)\right\} \varepsilon^{2 \alpha}
\end{aligned}
$$

$$
\begin{aligned}
& +\left\{\frac{(1+a \eta)^{4 \alpha}}{\Gamma(1+4 \alpha)} \frac{\partial^{4 \alpha}}{\partial x^{4 \alpha}} \frac{\partial^{\alpha}}{\partial t^{\alpha}} f\left(\varphi_{0}\right)\right. \\
& +a \frac{\partial^{\alpha} f\left(\varphi_{0}\right)}{\partial x^{\alpha}} \frac{(1+a \eta)^{4 \alpha}}{\Gamma(1+4 \alpha)} \frac{\partial^{5 \alpha}}{\partial x^{5 \alpha}} f\left(\varphi_{0}\right) \\
& +\frac{a}{2}\left(\frac{(1+a \eta)^{2 \alpha}}{\Gamma(1+2 \alpha)} \frac{\partial^{3 \alpha}}{\partial x^{3 \alpha}} f\left(\varphi_{0}\right)\right)^{2}-a \frac{(1+a \eta)^{\alpha}}{\Gamma(1+\alpha)} \frac{\partial^{2 \alpha}}{\partial x^{2 \alpha}} \\
& \left.\quad \times f\left(\varphi_{0}\right) \frac{(1+a \eta)^{3 \alpha}}{\Gamma(1+3 \alpha)} \frac{\partial^{4 \alpha}}{\partial x^{4 \alpha}} f\left(\varphi_{0}\right)\right\} \varepsilon^{4 \alpha} \\
& +\eta+O\left(\varepsilon^{4 \alpha}\right)=0 .
\end{aligned}
$$

To this end, let us consider the following relations:

$$
\begin{aligned}
\varepsilon & =\frac{h}{\lambda} \ll 1, \\
a & =\frac{\varsigma}{h^{\alpha}} \ll 1 .
\end{aligned}
$$

Then, from (99) and (103), we have

$$
\begin{gathered}
\frac{\partial^{\alpha} \eta}{\partial t^{\alpha}}+\left(a \frac{\partial^{\alpha} f\left(\varphi_{0}\right)}{\partial x^{\alpha}} \frac{\partial^{\alpha} \eta}{\partial x^{\alpha}}+\frac{(1+a \eta)^{\alpha}}{\Gamma(1+\alpha)} \frac{\partial^{2 \alpha}}{\partial x^{2 \alpha}} f\left(\varphi_{0}\right)\right) \\
-\left\{\frac{(1+a \eta)^{2 \alpha}}{\Gamma(1+2 \alpha)} \frac{\partial^{3 \alpha}}{\partial x^{3 \alpha}} f\left(\varphi_{0}\right) a \frac{\partial^{\alpha} \eta}{\partial x^{\alpha}}\right. \\
\left.+\frac{(1+a \eta)^{3 \alpha}}{\Gamma(1+3 \alpha)} \frac{\partial^{4 \alpha}}{\partial x^{4 \alpha}} f\left(\varphi_{0}\right)\right\} \varepsilon^{2 \alpha}+O\left(\varepsilon^{4 \alpha}\right)=0, \\
\frac{\partial^{\alpha} f\left(\varphi_{0}\right)}{\partial t^{\alpha}}+\frac{a}{2}\left(\frac{\partial^{\alpha} f\left(\varphi_{0}\right)}{\partial x^{\alpha}}\right)^{2}-\frac{(1+a \eta)^{2 \alpha}}{\Gamma(1+2 \alpha)}
\end{gathered}
$$




$$
\begin{aligned}
& \times\left\{\frac{\partial^{2 \alpha}}{\partial x^{3 \alpha}} \frac{\partial^{\alpha} f\left(\varphi_{0}\right)}{\partial t^{\alpha}}+a \frac{\partial^{\alpha} f\left(\varphi_{0}\right)}{\partial x^{\alpha}} \frac{\partial^{3 \alpha} f\left(\varphi_{0}\right)}{\partial x^{3 \alpha}}\right. \\
& \left.-\frac{a}{2} \frac{\Gamma(1+2 \alpha)}{\Gamma^{2}(1+\alpha)}\left(\frac{\partial^{2 \alpha}}{\partial x^{2 \alpha}} f\left(\varphi_{0}\right)\right)^{2}\right\} \varepsilon^{2 \alpha} \\
& +\eta+O\left(\varepsilon^{4 \alpha}\right)=0 .
\end{aligned}
$$

From (101), we get

$$
\begin{gathered}
\frac{\partial^{\alpha} \eta}{\partial t^{\alpha}}+a \frac{\partial^{\alpha} f\left(\varphi_{0}\right)}{\partial x^{\alpha}} \frac{\partial^{\alpha} \eta}{\partial x^{\alpha}}+\frac{(1+a \eta)^{\alpha}}{\Gamma(1+\alpha)} \frac{\partial^{2 \alpha}}{\partial x^{2 \alpha}} f\left(\varphi_{0}\right)=0, \\
\frac{\partial^{\alpha} f\left(\varphi_{0}\right)}{\partial t^{\alpha}}+\frac{a}{2}\left(\frac{\partial^{\alpha} f\left(\varphi_{0}\right)}{\partial x^{\alpha}}\right)^{2}+\eta=0
\end{gathered}
$$

which yield

$$
\begin{gathered}
\frac{\partial^{\alpha} \eta}{\partial t^{\alpha}}+a \sigma \frac{\partial^{\alpha} \eta}{\partial x^{\alpha}}+\frac{(1+a \eta)^{\alpha}}{\Gamma(1+\alpha)} \frac{\partial^{\alpha} \sigma}{\partial x^{\alpha}}=0, \\
\frac{\partial^{\alpha} \sigma}{\partial t^{\alpha}}+a \sigma \frac{\partial^{\alpha} \sigma}{\partial x^{\alpha}}+\frac{\partial^{\alpha} \eta}{\partial x^{\alpha}}=0,
\end{gathered}
$$

where

$$
\sigma=\frac{\partial^{\alpha} f\left(\varphi_{0}\right)}{\partial x^{\alpha}} .
$$

If the terms of $O\left(a \varepsilon^{2 \alpha}, \varepsilon^{4 \alpha}\right)$ are omitted, then from (101), we obtain

$$
\begin{aligned}
& \frac{\partial^{\alpha} \eta}{\partial t^{\alpha}}+ a \sigma \frac{\partial^{\alpha} \eta}{\partial x^{\alpha}}+\frac{(1+a \eta)^{\alpha}}{\Gamma(1+\alpha)} \frac{\partial^{\alpha} \sigma}{\partial x^{\alpha}}-\frac{(1+a \eta)^{3 \alpha}}{\Gamma(1+3 \alpha)} \frac{\partial^{3 \alpha}}{\partial x^{3 \alpha}} \sigma \varepsilon^{2 \alpha} \\
&+O\left(a \varepsilon^{2 \alpha}, \varepsilon^{4 \alpha}\right)=0 \\
& \frac{\partial^{\alpha} \sigma}{\partial t^{\alpha}}+a \sigma \frac{\partial^{\alpha} \sigma}{\partial x^{\alpha}}-\frac{(1+a \eta)^{2 \alpha}}{\Gamma(1+2 \alpha)} \frac{\partial^{2 \alpha}}{\partial x^{2 \alpha}} \frac{\partial^{\alpha} \sigma}{\partial t^{\alpha}}+\frac{\partial^{\alpha} \eta}{\partial x^{\alpha}} \\
&+O\left(a \varepsilon^{2 \alpha}, \varepsilon^{4 \alpha}\right)=0
\end{aligned}
$$

From (93) and (95), we have

$$
\begin{gathered}
\frac{\partial^{\alpha} \varphi}{\partial x^{\alpha}}=\sigma-\frac{1}{\Gamma(1+2 \alpha)} \frac{\partial^{2 \alpha}}{\partial x^{2 \alpha}} \sigma \varepsilon^{2 \alpha}+O\left(\varepsilon^{4 \alpha}\right), \\
\omega=\sigma-\frac{1}{\Gamma(1+2 \alpha)} \frac{\partial^{2 \alpha}}{\partial x^{2 \alpha}} \sigma \varepsilon^{2 \alpha}+O\left(a \varepsilon^{2 \alpha}, \varepsilon^{4 \alpha}\right),
\end{gathered}
$$

which can alternatively be written as

$$
\sigma=\omega+\frac{1}{\Gamma(1+2 \alpha)} \frac{\partial^{2 \alpha} \omega}{\partial x^{2 \alpha}} \varepsilon^{2 \alpha}+O\left(a \varepsilon^{2 \alpha}, \varepsilon^{4 \alpha}\right) .
$$

Substituting (108) in (97) and (106), we get

$$
\begin{gathered}
\frac{\partial^{\alpha} \eta}{\partial t^{\alpha}}+a \omega \frac{\partial^{\alpha} \eta}{\partial x^{\alpha}}+\frac{1}{\Gamma(1+\alpha)} \frac{\partial^{\alpha} \omega}{\partial x^{\alpha}}+O\left(a \varepsilon^{2 \alpha}, \varepsilon^{4 \alpha}\right)=0, \\
\frac{\partial^{\alpha} \omega}{\partial t^{\alpha}}+a \omega \frac{\partial^{\alpha} \omega}{\partial x^{\alpha}}-\frac{1}{\Gamma(1+2 \alpha)} \frac{\partial^{2 \alpha}}{\partial x^{2 \alpha}} \frac{\partial^{\alpha} \omega}{\partial t^{\alpha}} \varepsilon^{2 \alpha} \\
+\frac{\partial^{\alpha} \eta}{\partial x^{\alpha}}+O\left(a \varepsilon^{2 \alpha}, \varepsilon^{4 \alpha}\right)=0,
\end{gathered}
$$

where $\varepsilon=h / \lambda \ll 1$ and $a=\varsigma / h^{\alpha} \ll 1$.

In view of (109), we obtain

$$
\begin{gathered}
\frac{\partial^{\alpha} \eta}{\partial t^{\alpha}}+\frac{1}{\Gamma(1+\alpha)} \frac{\partial^{\alpha} \omega}{\partial x^{\alpha}}=0, \\
\frac{\partial^{\alpha} \omega}{\partial t^{\alpha}}+\frac{\partial^{\alpha} \eta}{\partial x^{\alpha}}=0,
\end{gathered}
$$

which result into the form

$$
\frac{\partial^{2 \alpha} \omega}{\partial t^{2 \alpha}}=\frac{1}{\Gamma(1+\alpha)} \frac{\partial^{2 \alpha} \eta}{\partial x^{2 \alpha}} .
$$

We notice that (111) is the linear local fractional wave equation for water waves when $\omega=\eta$.

We may also transform (110) into the following forms:

$$
\begin{aligned}
& \frac{\partial^{\alpha} \eta}{\partial t^{\alpha}}+\frac{\partial^{\alpha} \omega}{\partial x^{\alpha}}=0, \\
& \frac{\partial^{\alpha} \omega}{\partial t^{\alpha}}+\frac{\partial^{\alpha} \eta}{\partial x^{\alpha}}=0,
\end{aligned}
$$

which yield

$$
\frac{\partial^{\alpha} \eta}{\partial t^{\alpha}}+\frac{\partial^{\alpha} \eta}{\partial x^{\alpha}}=0
$$

where $\omega=\eta$ and $\alpha \rightarrow 1$.

For developing the problem further, by (113), (105), and (106) can be expressed as

$$
\begin{aligned}
& \frac{\partial^{\alpha} \eta}{\partial t^{\alpha}}+a \sigma \frac{\partial^{\alpha} \eta}{\partial x^{\alpha}}+\frac{\partial^{\alpha} \sigma}{\partial x^{\alpha}}-\frac{1}{\Gamma(1+3 \alpha)} \frac{\partial^{3 \alpha}}{\partial x^{3 \alpha}} \sigma \varepsilon^{2 \alpha} \\
&+O\left(a \varepsilon^{2 \alpha}, \varepsilon^{4 \alpha}\right)=0, \\
& \frac{\partial^{\alpha} \sigma}{\partial t^{\alpha}}+a \sigma \frac{\partial^{\alpha} \sigma}{\partial x^{\alpha}}-\frac{1}{\Gamma(1+2 \alpha)} \frac{\partial^{2 \alpha}}{\partial x^{2 \alpha}} \frac{\partial^{\alpha} \sigma}{\partial t^{\alpha}}+\frac{\partial^{\alpha} \eta}{\partial x^{\alpha}} \\
&+O\left(a \varepsilon^{2 \alpha}, \varepsilon^{4 \alpha}\right)=0
\end{aligned}
$$

which lead to

$$
\begin{aligned}
& \frac{\partial^{\alpha} \eta}{\partial t^{\alpha}}+a \sigma \frac{\partial^{\alpha} \eta}{\partial x^{\alpha}}+\frac{\partial^{\alpha} \sigma}{\partial x^{\alpha}}-\frac{1}{\Gamma(1+3 \alpha)} \frac{\partial^{3 \alpha}}{\partial x^{3 \alpha}} \sigma \varepsilon^{2 \alpha}=0, \\
& \frac{\partial^{\alpha} \sigma}{\partial t^{\alpha}}+a \sigma \frac{\partial^{\alpha} \sigma}{\partial x^{\alpha}}-\frac{1}{\Gamma(1+2 \alpha)} \frac{\partial^{2 \alpha}}{\partial x^{2 \alpha}} \frac{\partial^{\alpha} \sigma}{\partial t^{\alpha}}+\frac{\partial^{\alpha} \eta}{\partial x^{\alpha}}=0,
\end{aligned}
$$

where $\varepsilon=h / \lambda \ll 1$ and $a=\varsigma / h^{\alpha} \ll 1$. 
In view of (108), (115), and (116), we obtain

$$
\begin{gathered}
\frac{\partial^{\alpha} \eta}{\partial t^{\alpha}}+a \omega \frac{\partial^{\alpha} \eta}{\partial x^{\alpha}}+\frac{\partial^{\alpha} \omega}{\partial x^{\alpha}}=0 \\
\frac{\partial^{\alpha} \omega}{\partial t^{\alpha}}+a \omega \frac{\partial^{\alpha} \omega}{\partial x^{\alpha}}-\frac{1}{\Gamma(1+2 \alpha)} \frac{\partial^{2 \alpha}}{\partial x^{2 \alpha}} \frac{\partial^{\alpha} \omega}{\partial t^{\alpha}} \varepsilon^{2 \alpha}+\frac{\partial^{\alpha} \eta}{\partial x^{\alpha}}=0 .
\end{gathered}
$$

Now, from (113), we may look for a solution of the form

$$
\sigma=\eta+a A+\varepsilon^{2 \alpha} B+O\left(a^{2}+\varepsilon^{4 \alpha}\right),
$$

where $A$ and $B$ are functions of $\eta$ and its local fractional derivatives.

Utilizing (115), (116), and (118), we obtain

$$
\begin{aligned}
\frac{\partial^{\alpha} \eta}{\partial t^{\alpha}}+ & a\left(\frac{\partial^{\alpha} A}{\partial x^{\alpha}}+\eta \frac{\partial^{\alpha} \eta}{\partial x^{\alpha}}\right)+\varepsilon^{2 \alpha}\left(\frac{\partial^{\alpha} B}{\partial x^{\alpha}}-\frac{1}{\Gamma(1+3 \alpha)} \frac{\partial^{3 \alpha}}{\partial x^{3 \alpha}} \eta\right) \\
+ & \frac{\partial^{\alpha} \eta}{\partial x^{\alpha}}+O\left(a^{2}+\varepsilon^{4 \alpha}\right)=0,
\end{aligned}
$$$$
\frac{\partial^{\alpha} \eta}{\partial t^{\alpha}}+a\left(\frac{\partial^{\alpha} A}{\partial t^{\alpha}}+\eta \frac{\partial^{\alpha} \eta}{\partial t^{\alpha}}\right)
$$$$
+\varepsilon^{2 \alpha}\left(\frac{\partial^{\alpha} B}{\partial t^{\alpha}}-\frac{1}{\Gamma(1+2 \alpha)} \frac{\partial^{2 \alpha}}{\partial x^{2 \alpha}} \frac{\partial^{\alpha} \sigma}{\partial t^{\alpha}}\right)
$$$$
+\frac{\partial^{\alpha} \eta}{\partial x^{\alpha}}+O\left(a^{2}+\varepsilon^{4 \alpha}\right)=0
$$

which lead to

$$
\begin{aligned}
\frac{\partial^{\alpha} \eta}{\partial t^{\alpha}}+ & a\left(\frac{\partial^{\alpha} A}{\partial x^{\alpha}}+\eta \frac{\partial^{\alpha} \eta}{\partial x^{\alpha}}\right)+\varepsilon^{2 \alpha}\left(\frac{\partial^{\alpha} B}{\partial x^{\alpha}}-\frac{1}{\Gamma(1+3 \alpha)} \frac{\partial^{3 \alpha} \eta}{\partial x^{3 \alpha}}\right) \\
& +\frac{\partial^{\alpha} \eta}{\partial x^{\alpha}}=0, \\
\frac{\partial^{\alpha} \eta}{\partial t^{\alpha}}+a\left(\frac{\partial^{\alpha} A}{\partial t^{\alpha}}+\eta \frac{\partial^{\alpha} \eta}{\partial t^{\alpha}}\right) & \\
& +\varepsilon^{2 \alpha}\left(\frac{\partial^{\alpha} B}{\partial t^{\alpha}}-\frac{1}{\Gamma(1+2 \alpha)} \frac{\partial^{2 \alpha}}{\partial x^{2 \alpha}} \frac{\partial^{\alpha} \sigma}{\partial t^{\alpha}}\right) \\
& +\frac{\partial^{\alpha} \eta}{\partial x^{\alpha}}=0 .
\end{aligned}
$$

Moreover, from (121), we have

$$
\begin{aligned}
& A=-\chi \eta^{2 \alpha}, \\
& B=\vartheta \frac{\partial^{2 \alpha} \eta}{\partial x^{2 \alpha}},
\end{aligned}
$$

such that (119) and (120) are valid with $\chi$ and $\vartheta$ as constants.

Therefore, from (115), (119), and (122), we have

$$
\sigma=\eta-a \chi \eta^{2 \alpha}+\varepsilon^{2 \alpha} \vartheta \frac{\partial^{2 \alpha} \eta}{\partial x^{2 \alpha}}+O\left(a^{2}+\varepsilon^{4 \alpha}\right),
$$

such that

$$
\frac{\partial^{\alpha} \eta}{\partial t^{\alpha}}-a M \eta \frac{\partial^{\alpha} \eta}{\partial x^{\alpha}}+\varepsilon^{2 \alpha} N \frac{\partial^{3 \alpha} \eta}{\partial x^{3 \alpha}}+\frac{\partial^{\alpha} \eta}{\partial x^{\alpha}}=0,
$$

where

$$
M=2 \chi-1 \neq 0
$$

$$
N=\vartheta-\frac{1}{\Gamma(1+3 \alpha)} \neq 0 \text {. }
$$

From (124), we arrive at the following formula:

$$
\frac{\partial^{\alpha} \eta}{\partial t^{\alpha}}-R \eta \frac{\partial^{\alpha} \eta}{\partial x^{\alpha}}+S \frac{\partial^{3 \alpha} \eta}{\partial x^{3 \alpha}}+\frac{\partial^{\alpha} \eta}{\partial x^{\alpha}}=0,
$$

which leads to

$$
\frac{\partial^{\alpha} \eta}{\partial t^{\alpha}}-\eta \frac{\partial^{\alpha} \eta}{\partial x^{\alpha}}+\frac{\partial^{3 \alpha} \eta}{\partial x^{3 \alpha}}+\frac{\partial^{\alpha} \eta}{\partial x^{\alpha}}=0,
$$

or (neglecting the low term)

$$
\frac{\partial^{\alpha} \eta}{\partial t^{\alpha}}-R \eta \frac{\partial^{\alpha} \eta}{\partial x^{\alpha}}+S \frac{\partial^{3 \alpha} \eta}{\partial x^{3 \alpha}}=0,
$$

where $\eta(x, t)$ is a nondifferentiable function, $R=a M$ and $S=\varepsilon^{2 \alpha} N$.

We notice that (126) is the local fractional Kortewegde Vries equation. When there are coefficient relations, namely, $R=1$ and $S=1$, we obtain a new local fractional Korteweg-de Vries equation. When neglecting the nonlinear term of (127), we obtain the linear local fractional Kortewegde Vries equation as follows:

$$
\frac{\partial^{\alpha} \eta}{\partial t^{\alpha}}+\frac{\partial^{3 \alpha} \eta}{\partial x^{3 \alpha}}+\frac{\partial^{\alpha} \eta}{\partial x^{\alpha}}=0
$$

where $\eta(x, t)$ is a nondifferentiable function.

\section{Conclusions}

In this work, we have derived the local fractional Korteweg-de Vries equation related to fractal waves on shallow water surfaces from the local fractional calculus view point. The linear and nonlinear theories for fractal water wave are presented and the linear and nonlinear local fractional Korteweg-de Vries equations are also obtained.

\section{Conflict of Interests}

The authors declare that they have no conflict of interests regarding this paper.

\section{References}

[1] J. Boussinesq, Essai sur la Théorie des Eaux Courantes, vol. 23 of Mémoires Présentés par Divers Savants à l'Académie des Sciences de l'Institut National de France, Imprimerie Nationale, 1877. 
[2] D. J. Korteweg and G. de Vries, "XLI. On the change of form of long waves advancing in a rectangular canal, and on a new type of long stationary waves," The London, Edinburgh, and Dublin Philosophical Magazine and Journal of Science, vol. 39, no. 240, pp. 422-443, 1895.

[3] J. Canosa and J. Gazdag, "The Korteweg-de Vries-Burgers equation," Journal of Computational Physics, vol. 23, no. 4, pp. 393-403, 1977.

[4] J. P. Boyd, "Solitons from sine waves: analytical and numerical methods for non-integrable solitary and cnoidal waves," Physica D: Nonlinear Phenomena, vol. 21, no. 2-3, pp. 227-246, 1986.

[5] M. Tsutsumi, T. Mukasa, and R. Iino, "On the generalized Korteweg-de Vries equation," Proceedings of the Japan Academy, vol. 46, no. 9, pp. 921-925, 1970.

[6] R. Dodd and A. Fordy, "The prolongation structures of quasipolynomial flows," Proceedings of the Royal Society of London A: Mathematical and Physical Sciences, vol. 385, no. 1789, pp. 389429, 1983.

[7] F. Calogero and A. Degasperis, Spectral Transform and Solitons: Tools to Solve and Investigate Nonlinear Evolution Equations, North-Holland, New York, NY, USA, 1982.

[8] R. S. Johnson, "On the inverse scattering transform, the cylindrical Korteweg-de Vries equation and similarity solutions," Physics Letters A, vol. 72, no. 3, pp. 197-199, 1979.

[9] G. Segal, "The geometry of the KdV equation," International Journal of Modern Physics A, vol. 6, no. 16, pp. 2859-2869, 1991.

[10] M. Wadati, "Stochastic Korteweg-de Vries equation," Journal of the Physical Society of Japan, vol. 52, no. 8, pp. 2642-2648, 1983.

[11] R. M. Miura, "Korteweg-de Vries equation and generalizations. I. A remarkable explicit nonlinear transformation," Journal of Mathematical Physics, vol. 9, no. 8, pp. 1202-1204, 1968.

[12] J. W. Miles, "The Korteweg-de Vries equation: a historical essay," Journal of Fluid Mechanics, vol. 106, pp. 131-147, 1981.

[13] S. Momani, Z. Odibat, and A. Alawneh, "Variational iteration method for solving the space- and time-fractional KdV equation," Numerical Methods for Partial Differential Equations, vol. 24, no. 1, pp. 262-271, 2008.

[14] S. A. El-Wakil, E. Abulwafa, M. Zahran, and A. Mahmoud, "Time-fractional KdV equation: formulation and solution using variational methods," Nonlinear Dynamics, vol. 65, no. 1-2, pp. 55-63, 2011.

[15] A. Atangana and A. Secer, "The time-fractional coupledKorteweg-de-Vries equations," Abstract and Applied Analysis, vol. 2013, Article ID 947986, 8 pages, 2013.

[16] O. Abdulaziz, I. Hashim, and E. S. Ismail, "Approximate analytical solution to fractional modified KdV equations," Mathematical and Computer Modelling, vol. 49, no. 1-2, pp. 136145, 2009.

[17] X.-J. Yang, Advanced Local Fractional Calculus and Its Applications, World Science, New York, NY, USA, 2012.

[18] X.-J. Yang, D. Baleanu, and J.-H. He, "Transport equations in fractal porous media within fractional complex transform method," Proceedings of the Romanian Academy A, vol. 14, no. 4, pp. 287-292, 2013.

[19] Y.-J. Hao, H. M. Srivastava, H. Jafari, and X.-J. Yang, "Helmholtz and diffusion equations associated with local fractional derivative operators involving the Cantorian and Cantor-type cylindrical coordinates," Advances in Mathematical Physics, vol. 2013, Article ID 754248, 5 pages, 2013.

[20] Y. Zhao, D. Baleanu, C. Cattani, D. F. Cheng, and X.-J. Yang, "Maxwell's equations on Cantor sets: a local fractional approach," Advances in High Energy Physics, vol. 2013, Article ID 686371, 6 pages, 2013.

[21] X.-J. Yang, D. Baleanu, and J. A. Tenreiro Machado, "Systems of Navier-Stokes equations on cantor sets," Mathematical Problems in Engineering, vol. 2013, Article ID 769724, 8 pages, 2013.

[22] A. M. Yang, C. Cattani, C. Zhang, G. N. Xie, and X. J. Yang, "Local fractional Fourier series solutions for non-homogeneous heat equations arising in fractal heat flow with local fractional derivative," Advances in Mechanical Engineering, vol. 2014, Article ID 514639, 5 pages, 2014.

[23] Y. Y. Li, Y. Zhao, G. N. Xie, D. Baleanu, X. J. Yang, and K. Zhao, "Local fractional Poisson and Laplace equations with applications to electrostatics in fractal domain," Advances in Mathematical Physics, vol. 2014, Article ID 590574, 5 pages, 2014.

[24] C. Y. Long, Y. Zhao, and H. Jafari, "Mathematical models arising in the fractal forest gap via local fractional calculus," Abstract and Applied Analysis, vol. 2014, Article ID 782393, 6 pages, 2014.

[25] S. Q. Wang, Y. J. Yang, and H. K. Jassim, "Local fractional function decomposition method for solving inhomogeneous wave equations with local fractional derivative," Abstract and Applied Analysis, vol. 2014, Article ID 176395, 7 pages, 2014.

[26] X.-J. Yang, H. M. Srivastava, J.-H. He, and D. Baleanu, "Cantortype cylindrical-coordinate method for differential equations with local fractional derivatives," Physics Letters A, vol. 377, no. 28-30, pp. 1696-1700, 2013.

[27] A. A. Kilbas, H. M. Srivastava, and J. J. Trujillo, Theory and Applications of Fractional Differential Equations, vol. 204 of North-Holland Mathematical Studies, Elsevier, Amsterdam, The Netherlands, 2006.

[28] X.-J. Ma, H. M. Srivastava, D. Baleanu, and X.-J. Yang, "A new Neumann series method for solving a family of local fractional Fredholm and Volterra integral equations," Mathematical Problems in Engineering, vol. 2013, Article ID 325121, 6 pages, 2013.

[29] A.-M. Yang, Z.-S. Chen, H. M. Srivastava, and X.-J. Yang, "Application of the local fractional series expansion method and the variational iteration method to the Helmholtz equation involving local fractional derivative operators," Abstract and Applied Analysis, vol. 2013, Article ID 259125, 6 pages, 2013.

[30] W. Wei, H. M. Srivastava, L. Wang, P.-Y. Shen, and J. Zhang, "A local fractional integral inequality on fractal space analogous to Aderson's inequality," Abstract and Applied Analysis, vol. 2014, Article ID 797561, 7 pages, 2014. 


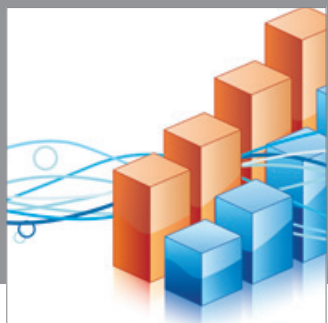

Advances in

Operations Research

mansans

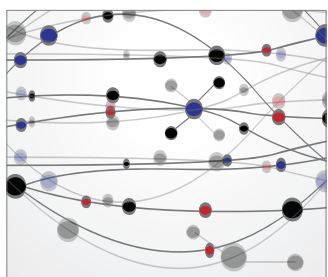

The Scientific World Journal
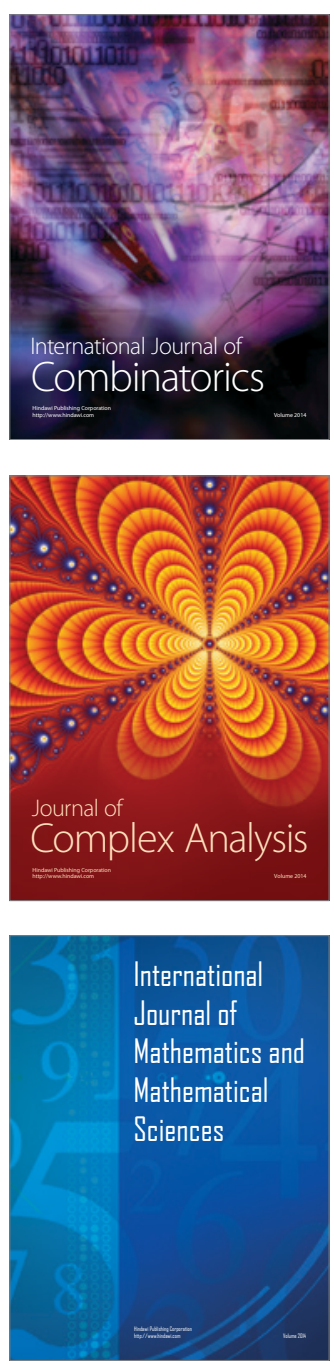
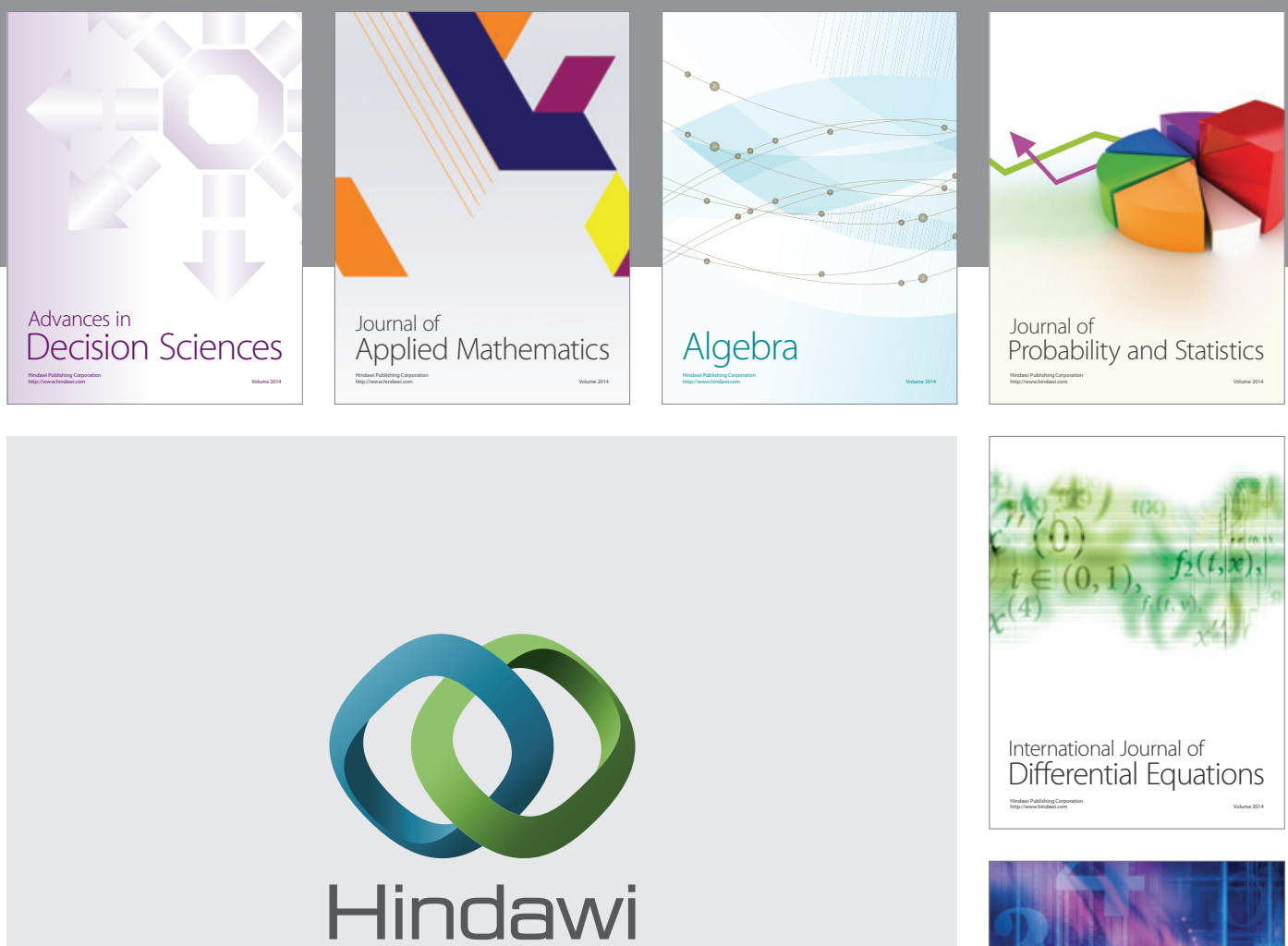

Submit your manuscripts at http://www.hindawi.com
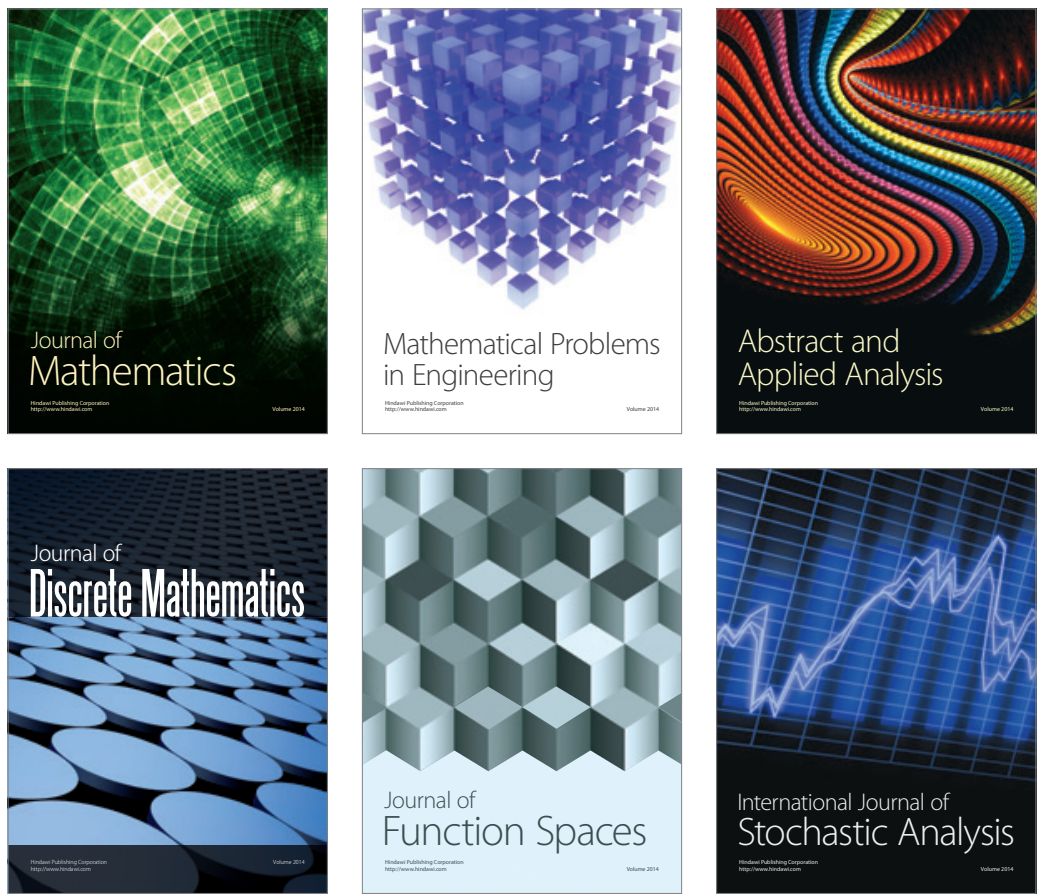

Journal of

Function Spaces

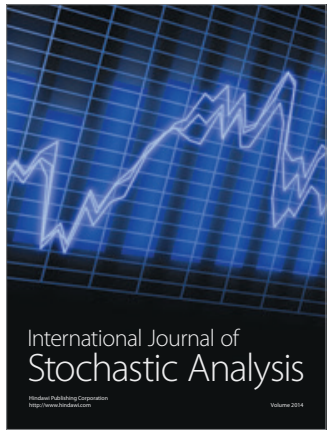

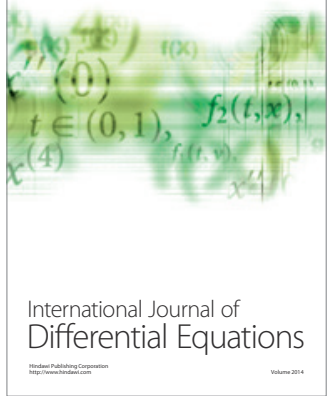
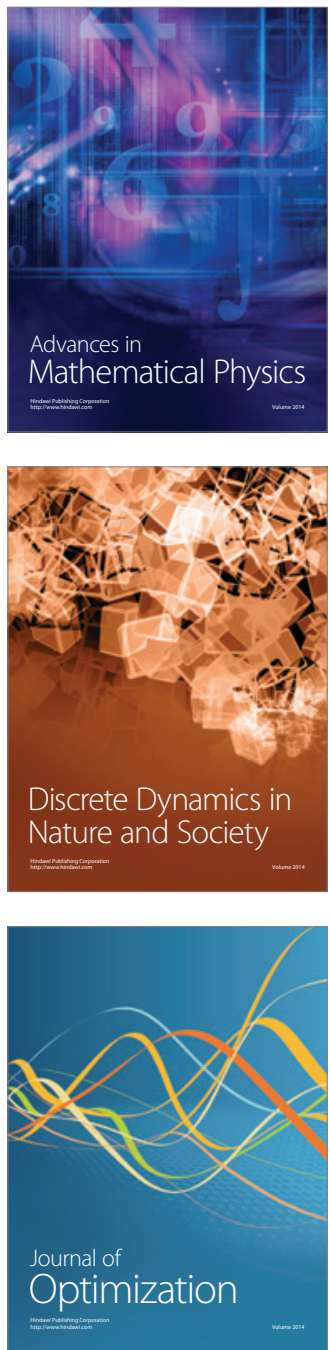